Maurer School of Law: Indiana University

Digital Repository @ Maurer Law

\title{
Racial Differences in the Mental Health Needs and Service Utilization of Youth in the Juvenile Justice System
}

\author{
Michael Jenuwine \\ Indiana University Maurer School of Law \\ Purva Rawal \\ Northwestern University Feinberg School of Medicine \\ Jill Romansky \\ Northwestern University Feinberg School of Medicine \\ John S. Lyons \\ Northwestern University Feinberg School of Medicine
}

Follow this and additional works at: https://www.repository.law.indiana.edu/facpub

Part of the Criminology and Criminal Justice Commons, Inequality and Stratification Commons, and the Juvenile Law Commons

\section{Recommended Citation}

Jenuwine, Michael; Rawal, Purva; Romansky, Jill; and Lyons, John S., "Racial Differences in the Mental Health Needs and Service Utilization of Youth in the Juvenile Justice System" (2004). Articles by Maurer Faculty. 2522.

https://www.repository.law.indiana.edu/facpub/2522

This Article is brought to you for free and open access by the Faculty Scholarship at Digital Repository @ Maurer Law. It has been accepted for inclusion in Articles by Maurer Faculty by an authorized administrator of Digital Repository @ Maurer Law. For more information, please contact rvaughan@indiana.edu. 


\title{
Racial Differences in the Mental Health Needs and Service Utilization of Youth in the Juvenile Justice System
}

\author{
Purva Rawal, BA \\ Jill Romansky, BS \\ Michael Jenuwine, PhD, JD \\ John S. Lyons, PhD
}

\begin{abstract}
Mental health placement rates by the juvenile justice system differ by race. However, it is unknown whether mental health needs differ by race. This study attempted to investigate potential differences in mental health needs and service utilization among Caucasian, African American, and Hispanic juvenile justice involved youth. A stratified random sample of 473 youth petitioned, adjudicated, and incarcerated from 1995-1996 was examined using a standard chart review protocol and the Childhood Severity of Psychiatric Illness measure for mental health needs. Significant and unique mental health needs were demonstrated for all racial groups. African American youth demonstrated the greatest level of needs. Minority status indicated significantly lower rates of mental health service utilization. Minority youth in the juvenile justice system are most at risk for underserved mental health needs. Based on the current data, it can be inferred that the first contact with the state's child and adolescent serving system, which includes the juvenile justice and mental health sectors, appears to be through the juvenile justice sector for many minority youth with delinquency problems.
\end{abstract}

\section{Introduction}

The juvenile crime rate has increased dramatically in the past 15 years, at rates estimated to be near $22 \% .^{1}$ Approximately 2 million youth are arrested in the United States each year and approximately 100,000 are placed in juvenile detention and correction facilities on each day. ${ }^{2}$ However, juvenile crime is far from just a contemporary problem. Since the creation of the first juvenile court in Illinois in 1899 , a rehabilitative approach has framed how we work with delinquent youth in the United

\footnotetext{
Address correspondence to Purva Rawal, BA, predoctoral fellow, Institute for Health Services Research and Policy Studies, and student, Division of Psychology and the Mental Health Services and Policy Program, Northwestern University Feinberg Medical School, 339 E Chicago Ave, Suite 717, Chicago, IL 60614. E-mail: p-rawal@ northwestern.edu.

Jill Romansky, BS, is a student in the Division of Psychology and the Mental Health Services and Policy Program and was a predoctoral fellow in the Institute for Health Services Research and Policy Studies, Northwestern University Feinberg Medical School, Chicago, Ill.

Michael Jenuwine, PhD, JD, is a Clinical Associate Professor of Law, and Associate Director, Child Advocacy Clinic at the Indiana University School of Law, Bloomington, Ind.

John S. Lyons, PhD, is the Director of the Mental Health Services and Policy Program in the Division of Psychology at the Northwestern University Feinberg Medical School, Chicago, Ill.
}

Journal of Behavioral Health Services \& Research, 2004, 31(3), 242-254. (C) 2004 National Council for Community Behavioral Healthcare. 
States. ${ }^{3}$ As noted in the Surgeon General's report, effectively identifying and addressing mental health needs in this high-risk population is an important priority for rehabilitation. ${ }^{4}$

One of the most striking findings in recent years is the high rate and wide range of diagnosable mental illness within the male and female juvenile justice systems in various samples. ${ }^{5-9}$ Approximately $20 \%$ of youth with histories of or current contact with the criminal justice system (eg, detention, probation) also meet diagnostic criteria for a diagnosable serious mental illness. ${ }^{10}$ Children and adolescents with a diagnosable serious mental illness are youth up to the age of 18 years who at any time over the past year have had or currently have a diagnosable mental, emotional, or behavioral disorder of sufficient duration to meet the Diagnostic and Statistical Manual of Mental Disorders, Third Edition, Revised criteria for significant school, family, or community functional impairment. ${ }^{11}$ Some estimates suggest that more than half of incarcerated youth have more than 1 diagnosable mental illness. ${ }^{8}$ The severity of mental illness in juvenile justice samples has been found to be similar to that of youth in residential treatment, one of the most restrictive out-of-home placements for children and adolescents, and in inpatient psychiatric hospitals. ${ }^{6,12,13}$

The literature indicates a significant degree of overlap between the mental health and juvenile justice systems, as well as the notable impact of mental health needs on the development and maintenance of delinquent behavior. ${ }^{2,4-9,14,15}$ Mental health needs, defined in the current study as a general need for mental health intervention to address problematic symptoms and/or behaviors, are often displayed in inappropriate conduct among children and adolescents. Similarly, youth come to the attention of law enforcement officials and eventually the juvenile justice system because of their outward behaviors. Other authors have discussed the "mad" versus "bad" distinction, outlining differences between those offenders whose unlawful behavior can be attributed to mental abnormalities versus those whose illegal acts are the result of moral corruption. ${ }^{16,17}$ It remains unclear what factors, such as the mental health characteristics of the youth, motivate a referral for mental health evaluation and services and what factors result in arrest and juvenile justice involvement. Thus, the precipitating factors for entry into the mental health system versus the juvenile justice system remain largely unknown.

Despite recent increased attention to youth with diagnosable mental illnesses within the juvenile justice system, there remains little epidemiological data on the prevalence of mental illness within minorities in this population. ${ }^{18}$ However, there are statistics on the percentage of minorities involved in the juvenile justice system. A report released by the US Department on Justice in 2000 stated that minorities comprise well over $20 \%$ of the overall juvenile justice system. More than $40 \%$ of arrests for violent crime and more than $25 \%$ for property crime involve African American Youth. ${ }^{1}$ A 1999 report released by the National Center for Juvenile Justice stated that minority involvement in juvenile crime is projected to increase substantially from 1995 to 2015, with a 19\% increase projected for African Americans compared to $3 \%$ for Caucasians. ${ }^{19}$ Given the substantial proportion of minorities and their projected increased involvement in the juvenile justice system, understanding the differential mental health needs and treatment experiences of minority youth should impact prevention efforts, identification, assessment, and the development of culturally competent mental health treatments.

Despite the significant rates of diagnosable mental illness within the juvenile justice system indicated in the literature, the probability of a given youth receiving a mental health placement (ie, community-based and institutional mental health interventions, such as outpatient or residential treatments) is estimated to be approximately $4 \%$, as demonstrated in a sample of 33,423 youth with contact with the juvenile courts between July 1, 1992, and June 30, 1994, in an East Coast state. ${ }^{20}$ The same sample yielded strong evidence indicating differential use of mental health placements depending on race and gender. Herz found that Caucasian females are as many as 8 times more likely to receive mental health placements than are African American males and 2.5 times more likely than are African American females and Caucasian males. ${ }^{20}$ Other evidence in the literature suggests that minority youth, including African Americans, Hispanics, and Asian Americans, are currently "underserved and/or inappropriately" served by the mental health and juvenile justice systems. ${ }^{18}$ 
Thus, several studies suggest that race may be one factor influencing mental health placement in juvenile justice or that minority youth may be less likely to receive mental health placements. It could also be the case that minority youth have differential mental health needs. However, the mental health needs of minority youth in the juvenile justice system are not well documented. ${ }^{7,21}$ The literature provides conflicting information. There is some indication that African American youth are more likely to experience psychological distress due to increased risks for violence in their home and neighborhood environments. ${ }^{21,22}$ Other studies have found no significant differences in mental health needs by race. ${ }^{23}$ However, these studies are often school based and do not comprise youth involved in the juvenile justice sector, specifically minorities, making it difficult to make conclusions about the level of mental health needs in minorities engaging in criminal behavior.

This study examines mental health needs among Caucasian, African American, and Hispanic youth in the juvenile justice system. The present study addresses the following 2 questions: are there identifiable and unique mental health needs for African American, Caucasian, and Hispanic youth involved in the juvenile justice system; and do juvenile justice involved youth of different races have different patterns of mental health services utilization? To address these questions, a stratified random sample of youth in the juvenile justice system was divided by race and then compared on various dimensions of symptoms, risk behaviors, functioning, comorbidity, caregiver capacity, utilization of mental health services, and demographic factors that have been shown to be different across racial categories.

\section{Methods}

A stratified random sample study design was used to ensure a representative juvenile justice sample of the state. Three counties were selected to represent urban, suburban, and rural areas of Illinois' juvenile justice system. Data were collected from these counties between 1995 and 1996, when the Illinois Juvenile Justice Commission authorized a study examining the mental health needs of juveniles.

Trained research assistants and one of the authors (J.S.L.) collected data. Identical assessment procedures were carried out at each site. Clinical data were gathered from case records using the Childhood Severity of Psychiatric Illness (CSPI) Scale, a reliable and well-validated instrument that assesses the mental health needs of children and adolescents on a variety of dimensions, including symptoms, risk behaviors, functioning, comorbidity, caregiver capacity, and multisystem needs. ${ }^{24-28}$ Reliability following training has been reported to exceed 0.80 in other retrospective file review studies. ${ }^{24,25}$ Audit reliability (comparison of prospectively completed CSPIs to those completed on the same children retrospectively) is above $0.70 .{ }^{27}$ Concurrent validity has been demonstrated through correlational comparisons with the Child and Adolescent Functional Assessment Scale (CAFAS) and the Child Behavior Checklist. ${ }^{24}$ Predictive validity has been demonstrated through the relationship of CPSI profiles to level of care decision making. ${ }^{24,25,28}$ Thus, the CSPI has been used extensively in child and adolescent serving agencies and demonstrated to be accurate and informative in assessing clinical status and needs of youth using retrospective file review methods. ${ }^{25,26}$

The CSPI uses a 4-point anchor scale for each item (eg, emotional disturbance, danger to self, intellectual functioning, adjustment problems): 0 indicates no problem or history of problem; 1 , mild problem of history or problem; 2, moderate problem; and 3, severe problem. Mental health needs in this study are defined based on CSPI dimension ratings. An example of one CSPI dimension is provided in Table 1.

In the current study, the CSPI was completed based on juvenile court and case records rated at the youth's most recent entry into the juvenile justice system. Reliability of the CSPI ratings among raters was 0.81 . In addition to the CSPI, demographic, social, and service history data were collected from the juvenile court records and case records, using a standard research protocol. All reviewers were trained to a reliability of 0.80 and reliability was monitored using selected cases throughout the study. The average reliability of selected cases was 0.87 . 


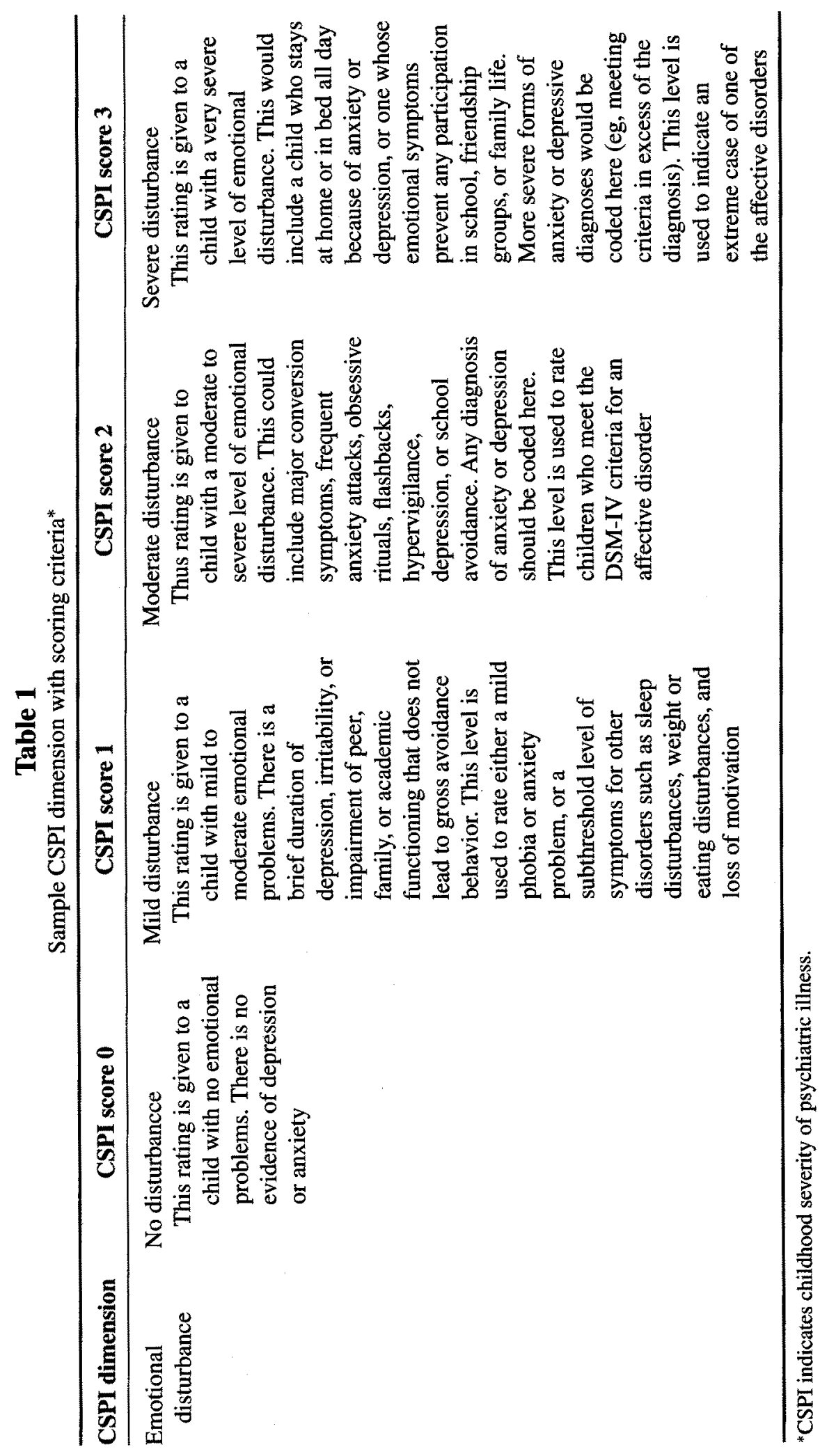




\section{Sample characteristics}

A total of 473 petitioned, adjudicated, and incarcerated cases were reviewed. The sample size of 450 was targeted and oversampling was used in case some sampled case records could not be located. A sample of 450 allowed for a confidence interval of $\pm 4 \%$ for most estimates. The mean age of the sample was 15.2 years $(\mathrm{SD}=1.5$; range $=8.3-18.0)$. The majority of the sample was male, with $83.3 \%(n=394)$ males and $16.1 \%(n=76)$ females. The sex for $0.6 \%(n=3)$ of the cases was unspecified.The sample was racially diverse, with $34.2 \%$ being African American $(n=$ $162), 29.8 \%$ Caucasian $(n=141), 14.2 \%$ Hispanic $(n=67), 3.0 \%$ Native American $(n=14), 2.7 \%$ Asian or "other" ( $n=13)$, and $18.8 \%(n=89)$ for whom the race was unspecified. Because of the low numbers of Native American and Asian or "other" youth, any statistical conclusions based on these categories were deemed unreliable; thus, only African American, Caucasian, and Hispanic youth were included in the sample, which totaled 370 cases. The mean age of the final sample was 15.2 years $(\mathrm{SD}=1.5$; range $=8.3-18.0$ ). The mean age in the sample indicates an older adolescent sample; thus, the terms youth and adolescent refer to older adolescents. The majority of the final sample was also male, with $82.7 \%$ males $(n=306), 16.8 \%$ females $(n=62)$, and $0.5 \%$ of cases unspecified $(n=2)$. The final sample comprised $38.1 \%$ Caucasian youth $(n=141), 43.8 \%$ African American youth $(n=162)$, and $18.1 \%$ Hispanic youth $(n=67)$.

\section{Statistical methods}

The Statistical Package for the Social Sciences (SPSS) version 10.1 was used to analyze all data. The primary method of analyses was 1-way analysis of variance (ANOVA) with post hoc Bonferroni tests to reduce the likelihood of a Type I error for detecting racial differences in symptoms, functioning, comorbidities, and caregiver capacity. Chi-square tests were also conducted to detect demographic and mental health treatment history differences among the 3 racial groups.

\section{Results}

\section{Demographic comparisons}

Demographic comparisons among the 3 groups are shown in Table 2. Sex of subject was significantly different across groups, with Caucasian youth having the highest percentage of females, followed by African American youth, and then Hispanic youth.

Hispanic and Caucasian youth were more likely than African American youth to have been in custody of at least one biological parent at the time of judicial petition. African American youth were more likely than the other 2 groups to have been in custody of other relatives at the time of petition. While biological mothers were present in the household at the time of petition in the majority of cases, biological fathers were present in the household in less than half of the cases. Hispanic youth were most likely to have had a biological mother or a father living in the household, followed by Caucasian youth, and then African American youth.

The percentage of African American youth with prior physical abuse, sexual abuse, or neglect histories was twice as high as that of Caucasian and Hispanic youth. Caucasian youth were more likely to have evidenced moderate-to-severe alcohol abuse problems and to have been in prior outpatient substance abuse treatment, followed by African American, and then Hispanic youth. The 3 groups did not significantly differ with regard to drug abuse problems.

\section{Mental health needs}

Results of the ANOVA and post hoc analyses can be seen in Table 3. 
Table 2

Comparison of African American, Caucasian, and Hispanic samples on demographic characteristics*

\begin{tabular}{|c|c|c|c|c|c|}
\hline \multirow[b]{2}{*}{ Characteristics } & \multicolumn{3}{|c|}{$\%$} & \multirow[b]{2}{*}{$\boldsymbol{n}$} & \multirow[b]{2}{*}{$\chi^{2}$} \\
\hline & $\begin{array}{l}\text { African } \\
\text { American }\end{array}$ & Caucasian & Hispanic & & \\
\hline \multicolumn{6}{|l|}{ Sex } \\
\hline Male & 85 & 78 & 91 & 368 & $5.98^{\dagger}$ \\
\hline Case type & & & & & NS \\
\hline Detention admission & 56 & 50 & 55 & & \\
\hline Probation referral & 41 & 47 & 45 & & \\
\hline Secure placement IDOC & 3 & 3 & 0 & & \\
\hline Treatment facility & 0 & 1 & 0 & & \\
\hline Custody & & & & 353 & $16.31^{\dagger}$ \\
\hline Parents & 81 & 93 & 94. & & \\
\hline Relatives & 12 & 2 & 3 & & \\
\hline Adoptive parents & 1 & 1 & 0 & & \\
\hline Foster parents & 1 & 0 & 0 & & \\
\hline DCFS & 5 & 5 & 3 & & \\
\hline Biological mother in home & 71 & 85 & 91 & 362 & $15.47^{\ddagger}$ \\
\hline Biological father in home & 17 & 31 & 43 & 362 & $18.87^{+}$ \\
\hline Prior abuse/neglect & 23 & 12 & 10 & 339 & $8.28^{\dagger}$ \\
\hline Alcohol abuse & 19 & 33 & 15 & 370 & $12.56^{\S}$ \\
\hline Drug abuse & 36 & 37 & 30 & & NS \\
\hline $\begin{array}{l}\text { Prior outpatient substance } \\
\text { abuse treatment }\end{array}$ & 3 & 10 & 0 & 370 & $13.25^{\S}$ \\
\hline $\begin{array}{l}\text { Prior inpatient substance } \\
\text { abuse treatment }\end{array}$ & 1 & 4 & 0 & & NS \\
\hline $\begin{array}{l}\text { Prior outpatient mental } \\
\text { health treatment }\end{array}$ & 10 & 11 & 10 & & NS \\
\hline $\begin{array}{l}\text { Prior inpatient mental } \\
\text { health treatment }\end{array}$ & 9 & 9 & 3 & & NS \\
\hline Prior RTC & 1 & 1 & 0 & & NS \\
\hline
\end{tabular}

\section{Symptoms}

The Hispanic subgroup displayed significantly less emotional disturbance than did both the African American and Caucasian subgroups. The Caucasian subgroup was rated as significantly lower than the Hispanic subgroup on conduct behavior and significantly lower than the African American subgroup on oppositional behavior. The 3 subgroups did not significantly differ with regard to the other CSPI symptoms indices, on the basis of total CSPI symptoms. 
Table 3

Comparison of African American, Caucasian, and Hispanic sample means on CSPI indices*

\begin{tabular}{lcccc}
\hline CSPI index & African American & Caucasian & Hispanic & $\boldsymbol{F}$ \\
\hline Neuropsychiatric & 0.0062 & 0.0213 & 0.00 & 1.249 \\
Emotional & $0.52^{\mathrm{a}}$ & $0.53^{\mathrm{a}, \mathrm{b}}$ & $0.25^{\mathrm{c}}$ & $3.942^{\dagger}$ \\
Conduct disorder & 1.67 & $1.48^{\mathrm{a}}$ & $1.85^{\mathrm{b}}$ & $6.345^{\dagger}$ \\
Oppositional behavior & $1.40^{\mathrm{a}}$ & $1.13^{\mathrm{b}}$ & 1.28 & $4.329^{\dagger}$ \\
Impulsivity & 1.09 & 1.01 & 1.28 & 2.675 \\
Contextual consistency & 1.51 & 1.21 & 1.21 & 2.454 \\
Total symptoms & 4.9691 & 4.3617 & 4.806 & 2.290 \\
Suicide risk & $0.0432^{\mathrm{a}}$ & $0.13^{\mathrm{b}}$ & 0.0299 & $3.773^{\dagger}$ \\
Danger to others & 1.31 & 1.21 & 1.45 & 1.40 \\
Elopement risk & 0.23 & 0.35 & 0.13 & 2.417 \\
Crime delinquency & $2.20^{\mathrm{a}, \mathrm{c}}$ & $1.80^{\mathrm{b}}$ & $2.45^{\mathrm{c}}$ & $18.285^{\S}$ \\
Sexual aggression & 0.0802 & 0.13 & 0.0746 & 0.472 \\
Total risk behaviors & 1.6667 & 1.8227 & 1.6866 & 0.494 \\
School impairment & 1.81 & 1.56 & 1.69 & 1.816 \\
Family impairment & $1.17^{\mathrm{a}, \mathrm{b}}$ & $0.95^{\mathrm{b}}$ & $0.48^{\mathrm{c}}$ & $13.556^{\S}$ \\
Peer impairment & $1.75^{\mathrm{a}, \mathrm{c}}$ & $1.16^{\mathrm{b}}$ & $1.90^{\mathrm{c}}$ & $14.635^{\S}$ \\
Total functioning & $4.7284^{\mathrm{a}}$ & $3.6667^{\mathrm{b}}$ & 4.0597 & $6.910^{\ddagger}$ \\
Adjustment to trauma & 0.29 & 0.18 & 0.13 & 2.352 \\
Medical status & 0.16 & 0.0851 & 0.0746 & 1.760 \\
Substance abuse problems & 0.73 & 0.86 & 0.67 & 1.278 \\
Severity of abuse & 0.25 & 0.24 & 0.0896 & 2.263 \\
Sexual development & 0.21 & 0.23 & 0.10 & 0.939 \\
Learning disability & 0.48 & 0.31 & 0.30 & 2.895 \\
Total comorbidities & $2.1235^{\mathrm{a}}$ & 1.9149 & $1.3731^{\mathrm{b}}$ & $3.430^{\dagger}$ \\
Caregiver supervision & 1.07 & 0.86 & 0.90 & 1.869 \\
Caregiver motivation & $0.54^{\mathrm{a}}$ & 0.44 & $0.18^{\mathrm{b}}$ & $5.239^{\ddagger}$ \\
Caregiver knowledge & $0.59^{\mathrm{a}, \mathrm{b}}$ & $0.59^{\mathrm{b}}$ & $0.28^{\mathrm{c}}$ & $4.638^{\dagger}$ \\
Placement safety & $0.43^{\mathrm{a}}$ & $0.23^{\mathrm{b}}$ & 0.30 & $3.519^{\dagger}$ \\
Total caregiver problems & $2.6235^{\mathrm{a}}$ & 2.1206 & $1.6567^{\mathrm{b}}$ & $3.470^{\dagger}$ \\
Multisystem needs & $0.75^{\mathrm{a}, \mathrm{c}}$ & $0.55^{\mathrm{b}}$ & $0.84^{\mathrm{c}}$ & $7.250^{\ddagger}$ \\
Total CSPI & $20.5679^{\mathrm{a}}$ & $17.4397^{\mathrm{b}}$ & 18.0746 & $4.167^{\dagger}$ \\
\hline & & & &
\end{tabular}

*Post hoc comparisons are indicated by superscripts $\mathrm{a}, \mathrm{b}$, and $\mathrm{c}$.

${ }^{\dagger} P<.05$.

$\ddagger P<.01$.

$\S P<.001$.

\section{Risk factors}

The Caucasian subgroup presented significantly greater risk of suicide than did the African American subgroup. Although there were no significant differences in the crime/delinquency index between the African American and Hispanic youth, the Caucasian subgroup was rated as significantly lower than the other 2 groups on severity of crime/delinquency. The 3 did not significantly differ with regard to the other CSPI risk behaviors indices, on the basis of total CSPI risk behaviors. 


\section{Functioning}

The African American and Caucasian subgroups were not significantly different from one another on the index of family dysfunction, although the Hispanic youth evidenced significantly less family dysfunction than did the other 2 subgroups. The African American and Hispanic subgroups did not significantly differ on peer dysfunction, although the Caucasian youth demonstrated significantly less peer dysfunction than did the other 2 subgroups. The 3 did not differ significantly with regard to school dysfunction. The Caucasian youth were rated as having significantly lower total dysfunction than did the African American youth.

\section{Comorbidities}

The 3 subgroups did not significantly differ on specific measures of comorbidity, although the African American youth were significantly higher than the Hispanic youth on the total comorbidities index.

\section{Caregiver characteristics}

The caregivers of the African American subgroup demonstrated significantly less motivation to care for their youth than did the caregivers of the Hispanic subgroup and significantly less placement safety than did the caregivers of the Caucasian youth. In addition, the caregivers of the African American and Caucasian subgroups exhibited significantly less knowledge of the youth under their care than did the caregivers of the Hispanic subgroup. The African American subgroup was rated as having a greater severity of total caregiver characteristics as compared with the Hispanic subgroup. Finally, both the African American and Hispanic subgroups evidenced greater multisystem needs than did the Caucasian subgroup.

\section{Service utilization}

Mental health treatment history includes inpatient and outpatient substance abuse and mental health treatment, along with residential treatment. Because of the low incidence of inpatient mental health and residential treatment episodes, as well as the low incidence of substance abuse treatment overall and particularly for minority youth, the treatment variables were collapsed into the following categories: prior mental health or substance abuse treatment, current mental health or substance abuse treatment, and either current or prior mental health or substance abuse treatment.

See Table 4 for racial differences in service utilization, which were examined using prior and current treatment information. Caucasian youth had higher rates of prior, current, and overall mental

\section{Table 4}

Comparison of African American, Caucasian, and Hispanic youth on mental health services utilization

$\%$

\begin{tabular}{lccccc}
\cline { 2 - 5 } Characteristics & $\begin{array}{c}\text { African } \\
\text { American }\end{array}$ & Caucasian & Hispanic & $\boldsymbol{n}$ & $\boldsymbol{\chi}^{\mathbf{2}}$ \\
\hline Prior treatment history & 25.5 & 41.1 & 13.4 & 340 & $17.7^{*}$ \\
Currently in treatment & 20.9 & 41.9 & 13.4 & 339 & $23.0^{*}$ \\
Ever in treatment & 36.5 & 58.9 & 19.4 & 339 & $30.5^{*}$ \\
\hline
\end{tabular}

${ }^{*} P<0.05$. 
health treatment histories than did African American and Hispanic youth. Hispanic youth had the lowest rate of prior, current, and overall mental health service utilization, as compared with African American and Caucasian youth.

\section{Discussion}

Given the significant level of mental health needs and low prior service utilization, particularly among minority youth in the sample, it can be inferred that the juvenile justice system may be one of the first contacts with the state child and adolescent service system (ie, the juvenile justice and mental health sectors) for many of these youth. Results from the present study indicate identifiable and addressable mental health needs for youth in the juvenile justice system, thus supporting previous findings that youth involved in the juvenile justice system exhibit high rates and a wide range of diagnosable mental health illnesses. $2,5-9$

While mental health needs were apparent across the sample, differences in the types and severity of needs depending on race were evident. Caucasian youth were used as the reference group in this study because they were the majority racial group and this allowed for comparisons regarding potential racial differences. Overall, African American youth displayed the highest level of mental health needs compared to Caucasian and Hispanic youth. Furthermore, African American youth appear to be especially underserved, given their high rates of symptoms, functioning, and comorbidity needs and low rates of prior and current mental health service use. Despite having lower rates of overall need compared to African American youth, Caucasian youth had the highest rate of current and prior mental health service utilization, and Hispanic youth received the least amount of current and prior mental health treatment despite having considerable mental health needs.

In this sample, less African American youth were under parental custody compared to their Caucasian and Hispanic counterparts, had fewer biological parents residing in the home, experienced substantially higher rates of abuse and/or neglect, had lower levels of family, peer, and overall functioning, and had higher rates of caregiver problems on relevant CSPI dimensions. They also had the highest total CSPI score, indicating multiple needs. The high rates of neglect and abuse in this sample of juvenile justice involved in African American youth, as well as separation from family, are likely predisposing factors for depression, aggression, substance abuse among other problems. ${ }^{29}$

It should be noted that the subsample of Hispanic youth was smaller than that of the other 2 racial groups in this sample; therefore, interpretations of data are presented tentatively. In this sample, Hispanic youth were more likely than African American youth to have been under parental custody and more likely than both African American and Caucasian youth to have lived in the same household with a biological parent at the time of judicial petition. Mean ratings on System Factors CSPI variables indicate that, overall, caregivers of Hispanic youth were motivated for change and were generally knowledgeable about their children's psychological strengths and weaknesses. ${ }^{24}$ This caregiver capacity and involvement may reflect the particular importance and explicit role of family (ie, familismo) in Hispanic or Latino culture. ${ }^{30,31}$ In terms of the clinical needs of this sample, Hispanic youth were least likely to have evidenced alcohol abuse problems or emotional disturbance. Perhaps the family strengths addressed above serve as protective factors against such psychological distress. ${ }^{32}$

However, Hispanic youth in the sample displayed significantly more severe symptoms of conduct disorder and criminal delinquent behavior than did Caucasian youth, but had similar rates as did African American youth. Studies have documented a higher prevalence of disruptive behavior disorders among youth from minority groups, as compared to Caucasian youth. ${ }^{33}$ Such externalizing traits-antisocial behaviors, impulsivity, and oppositionality-likely lead to involvement in the juvenile justice system rather than the mental health service system. However, these symptoms still necessitate intervention. Capitalizing on the caregiver capacity to address the behavioral needs of Hispanic youth might represent a culturally competent intervention strategy. 
In this sample, Caucasian youth evidenced the highest rate of alcohol abuse problems and prior outpatient substance abuse treatment, as well as significantly greater suicide risk than did African American youth. Studies have documented a greater prevalence of substance abuse problems among Caucasian adults as compared with other racial groups. ${ }^{34,35}$ However, research to understand racial differences in substance abuse problems in adolescents must be conducted to support these findings, given the limited generalizability in extrapolating adult findings to adolescent literature. Previous research has also indicated an elevated risk of suicide for Caucasian versus African American youth. ${ }^{36}$

A number of hypotheses have been offered to explain the lower rate of mental health service utilization for African American youth. Studies have robustly demonstrated that African American adults are less likely to use mental health services. ${ }^{37,38}$ Evidence suggests that mental illness may carry a greater stigma among ethnic minority and lower-income populations. ${ }^{39-41}$ In addition, evidence suggests that African Americans are more likely to endorse supernatural or religious causes of mental illness as compared to other ethnic groups. ${ }^{39}$ Reasons for low utilization of mental health services for African American youth are less clear, although evidence suggests that adolescents, overall, are disinclined to seek help even after recognizing problems they are experiencing. ${ }^{42}$ Minority youth may be even less likely to seek mental health services because of cultural differences in acceptance of mental health treatment, although little is known specifically about cultural attitudes in adolescents since culture is often confounded with other variables such as socioeconomic status. ${ }^{42}$

Lower rates of service utilization may also be due to a lack of access to services and no insurance coverage or poor insurance coverage compared to that for Caucasian counterparts. ${ }^{21}$ Poverty and low income, which is of a high incidence in African American communities, also have been linked to an increased likelihood of having a diagnosable childhood or adolescent mental illness. ${ }^{43}$ Lastly, there may be racial bias in the system. There is evidence for stereotypes in the juvenile justice system, including findings that African American youth are viewed as more responsible for their criminal offending than are Caucasian youth. ${ }^{44}$ The difference between perceiving the actions of a youth as criminal (bad) rather than symptomatic of emotional difficulties (mad) may be determined in part by race. ${ }^{16,17,44}$ African American youth may be more likely to be viewed as conduct disordered and delinquent rather than as emotionally disturbed, whereas Caucasian youth may be more likely to be viewed as having a mental illness.

Hispanic youth in this sample had the lowest rate of mental health service utilization. Like African American youth, Hispanic youth likely encounter barriers in access to services. Latinos have been shown to have the lowest rate of health insurance coverage, impacted by low socioeconomic status and a high rate of immigration. ${ }^{45}$ A study of referrals to mental health services in the juvenile justice system found that being Latino was associated with not receiving a mental health referral; the authors cited evidence indicating that Latino parents may be more hesitant to seek mental health services for their children than are Caucasian parents. ${ }^{46,47}$ This could be due in part to negative views about mental illness and fear of stigma, as well as cultural beliefs about the causes of and proper means of treating mental illness. ${ }^{41,48}$ Furthermore, Hispanic or Latino caregivers and youth may seek assistance in dealing with psychological distress from family members or nonmental health specialists, like physicians and ministers/priests, rather than mental health professionals. ${ }^{30,31}$

While minority youth in this sample evidenced lower mental health service utilization than did Caucasian youth, we cannot conclude that the mental health needs of Caucasian youth are being adequately served. Although the Caucasian youth demonstrated significantly higher functioning than did African American youth, significant differences in overall comorbidity and caregiver needs were not established. Furthermore, given the high rate of psychiatric symptoms and substance abuse among the Caucasian youth, as well as the seemingly low rates of prior treatment history and the historical lack of mental health services for all juvenile justice-involved youth, Caucasian youth are probably underserved as well. Therefore, all youth, regardless of race, probably have underserved mental health needs. 
The present study had several limitations. The data were collected retrospectively, which may have resulted in chart biases and missing information, compromising the reliability of data collected. To counteract this limitation, a stratified random sample representative of the state juvenile justice system was used and tentative conclusions were offered. In addition, the significant level of mental health needs in this sample is comparable to the level of mental health needs found in interviewbased studies, in which over two thirds of males and three quarters of females in detention were found to have a diagnosable mental illness; this suggests that the current retrospective methodology yielded meaningful information similar to that found in prospective designs. ${ }^{49}$ However, the current study included youth outside of detention and had a broader definition of mental health needs that was not only related to a diagnosis, but also to the need for monitoring and intervention on symptom, risk behavior, functioning, comorbidity, and caregiver capacity and multisystem need dimensions. The analyses were conducted only by race and not also by geographic region for a number of reasons. First, the overall purpose of the study was to make conclusions based on a statewide sample. Second, although demographic differences exist between counties, the juvenile justice system operates consistently across the state. Third, there were concerns about sample size considerations and a loss of power if the sample was further broken up by region. Geographic analyses were not planned at the outset of the study; however, these analyses may be possible in an ongoing prospective data collection project. Furthermore, race and geography are difficult to tease out given that they may be correlated. Thus, with the current data, the focus remained on understanding statewide racial differences and analyses.

Although the overall sample was relatively large, the number of Hispanic youth $(n=67)$ was smaller than that of the other 2 racial categories, reflecting another limitation; therefore, conclusive statements about Hispanic youth cannot be made. The sample was overwhelmingly predominantly male as well, which limits the generalizability of the current study results to females; thus, future studies may want to oversample females in order to make conclusions about their specific mental health needs. In addition, the larger issue of developing a focus specifically for females in the juvenile justice system should be a priority for the research community.

Other significant limitations concern the exclusion of other ethnic/racial groups in the sample (eg, Native American, Asian American) and the use of broad ethnic/racial categories. The current study did not investigate within-group differences for the racial subgroups, which might be as or more relevant than differences found across global racial categories, particularly in more diverse urban settings. As such, future research must recognize and explore racial/ethnic variation within broader groups.

An additional limitation involved the collapsing together of substance abuse and mental health services in examining mental health services utilization. Doing so precludes the opportunity to make inferences about the differential use of mental health and substance abuse services between racial groups. However, this was done because of the low incidence of substance abuse treatment and also to increase the likelihood that any mental health or substance problem was addressed. Given the available data, analyses linking case types and other descriptors of juvenile justice history and mental health needs are not presented. Analyses of this kind would be helpful in further understanding specific differences in mental health needs between groups and also identifying points of intervention for service system failures. Such analyses are currently being conducted with a prospectively collected large statewide juvenile justice data set to address these issues. Data related to demographic variables, such as health insurance coverage and poverty, were generally discussed because the limitations of the study and its retrospective nature prevented collection of more specific information on many of these variables. Finally, this study does not address duration, quality, acceptance, or outcomes of mental health services. Service types (eg, residential, inpatient treatment) were combined because numbers of youth receiving the services were too small to make service type comparisons by race; thus, information on the differential use of services cannot be attained. 


\section{Implications for Behavioral Health Services}

Despite these limitations, this study indicates that there is an underutilization of services for juvenile justice youth with clear mental health needs. This study also identifies that differential mental health needs exist for racial groups represented in the juvenile justice system. Future research should continue to assess the nature of these needs and the availability of effective and culturally competent treatments to serve these needs. The types of mental health services warrant further investigation, as juvenile justice outcomes can be linked to the prevention of juvenile justice involvement and the return to the juvenile and adult criminal systems. Ultimately, outcomes data from multiple child serving systems, including mental health and juvenile justice, should be collected to determine whether these youth can be successfully identified and served in the community prior to their contact with the juvenile justice system.

\section{Acknowledgment}

This project was funded in part by the Illinois Juvenile Justice Commission. This work was completed while receiving funding through the National Research Service Award. The authors acknowledge Jane Barden for her assistance in the editing phase of this work.

\section{References}

1. Snyder HN. Office of Juvenile Justice and Delinquency Prevention: Juvenile Justice Bulletin. Washington, DC: US Dept of Justice; 2000.

2. National Mental Health Association. Justice for juveniles: how communities respond to mental health and substance abuse needs of youth in the juvenile justice system (executive Summary). 2000. Available at: http://www.nmha.org/children/justjuv/execsum.cfm. Accessed June 2, 2004

3. Addams J. Introduction to the Child, the Clinic and the Court. New York: New Republic Inc; 1925.

4. United States Public Health Service Report of the Surgeon General's Conference on Children's Mental Health: A National Action Agenda. Washington, DC: Office of Surgeon General; 2000.

5. McManus M, Alessi NE, Grapentine WL, et al. Psychiatric disturbance in serious delinquents. Journal of the American Academy of Child and Adolescent Psychiatry. 1984;23(5):602-615.

6. Davis DL, Bean GJ, Schumacher JE, et al. Prevalence of emotional disorders in a juvenile justice institutional population. American Journal of Forensic Psychology. 1991;9(1):5-17.

7. Wierson M, Forehand RL, Frame CL. Epidemiology and treatment of mental health problems in juvenile delinquents. Advanced Behavioral Research and Therapy. 1992;14:93-120.

8. Ulzen TP, Hamilton $H$. The nature and characteristics of psychiatric comorbidity in incarcerated adolescents. Canadian Joumal of $P_{s y c h i-}$ atry. 1998;43(1):57-63.

9. Kataoka SH, Zima BT, Dupre DA, et al, Mental health problems and service use among female juvenile offenders: their relationship to criminal history. Journal of the American Academy of Child and Adolescent Psychiatry. 2001;40(5):549-555.

10. American Psychiatric Association Committee on Nomenclature and Statistics. Diagnostic and Statistical Manual of Mental Disorders, Revised Third Edition. Washington, DC: American Psychiatric Association; 1997.

11. Costello EJ, Angold A, Burns BJ, et al. The Great Smoky Mountains Study of youth: functional impairment and serious emotional disturbance. Archives of General Psychiatry. 1996;53(12):1137-1143.

12. Lyons JS, Baerger DR, Quigley $P$, et al. Mental health service needs of juvenile offenders: a comparison of detention, incarceration, and treatment settings. Children's Services: Social Policy, Research, and Practice. 2001;4(2):69-85.

13. Cohen R, Parmelee DX, Irwin L, et al. Characteristics of children and adolescents in a psychiatric hospital and a corrections facility. Joumal of the American Academy of Child and Adolescent Psychiatry. 1990;35(4):500-507.

14. Rosenblatt JA, Rosenblatt A, Biggs EE. Criminal behavior and emotional disorder: comparing youth served by the mental health and juvenile justice systems. Joumal of Emotional and Behavioral Disorders. 2000;27(2):227-238.

15. McKinney K. OJJDP mental health initiatives. 2001. Available at: http://www.ncjrs.org/pdffiles1/ojjdp/fs200130.pdf. Accessed June 2, 2004.

16. Campbell $\mathrm{AD}$. Kansas v. Hendricks: absent a clear meaning of punishment, states are permitted to violate double jeopardy clause. Loyola University of Chicago Law Journal. 1998;30:87-131.

17. Steiker CS. Punishment and procedure: punishment theory and the criminal-civil procedural divide. Georgetown Law Journal. 1997;85:775819.

18. Isaacs MR. Assessing the mental health needs of children and adolescents of color in the juvenile justice system: overcoming institutionalized perceptions and barriers. In: Cocozza JJ, ed. Responding to the Mental Health Needs of Youth in the Juvenile Justice System. Seattle: National Coalition for the Mentally IIl in the Criminal Justice System; 1992:141-163.

19. Snyder HN, Sicklund M. Juvenile offenders and victims: 1999 National Report, National Center for Juvenile Justice. 1999. Available at: http://www.ncjrs.org/html/ojjdp/nationalreport99/chapter1.pdf. Accessed June 2, 2004. 
20. Herz DC. Understanding the use of mental health placements by the juvenile justice system. Journal of Emotional and Behavioral Disorders. $2001 ; 9(3): 172-181$.

21. Hoberman HM. Ethnic minority status and adolescent mental health services utilization. The Journal of Mental Health Administration. 1992;19(3):246-267.

22. Lewis DO, Balla DA, Shanok SS. Some evidence of race bias in the diagnosis and treatment of the juvenile offender. American Journal of Orthopsychiatry. 1978;49(1):53-61.

23. Casper RC, Belanoff J, Offer D. Gender differences, but no racial differences, in self-reported psychiatric symptoms in adolescents. Journal of the American Academy of Child and Adalescent Psychiatry. 1996;35(4):500-507.

24. Lyons IS. Severity and Acuity of Psychiatric Ilness manual: Child and Adolescent Version. San Antonio, Tex: The Psychological Corporation; 1998.

25. Lyons JS, Libman-Mintzer LN, Kisiel CL, et al. Understanding the mental health needs of children and adolescents in residential treatment. Professional Psychology: Research and Practice. 1998;29(6);582-587.

26. Lyons JS, Kisiel CL, Dulcan M, et al. Crisis assessment and psychiatric hospitalization of children and adolescents in state custody. Journal of Child and Family Studies. 1997:6:311-320.

27. Lyons IS, Rawal P, Yeh I, et al. Use of measurement audit in outcomes management. Journal of Behavioral Health Services \& Research. 2002;29:75-80.

28. Leon SC, Lyons JS, Uziel-Miller ND, et al. Evaluating the use of psychiatric hospitalization by residential treatment. Journal of American Academy of Child and Adolescent Psychiatry. 2001;39:1496-1501.

29. Kaplan SJ, Pelcovitz D, Salzinger S, et al. Adolescent physical abuse; risk for adolescent psychiatric disorders. American Journal of Psychiatry. 1998;155(7):954-959.

30. La Roche MJ. Culture, transference, and countertransference among Latinos. Psychotherapy. 1999;36(4):389-397.

31. La Roche MJ. Psychotherapeutic considerations in treating Latinos. Harvard Review of Psychiatry. 2002;10:115-122.

32. Steinberg L, Lamborn SD, Dornbusch SM, et al. Impact of parenting practices on adolescent achievement: authoritative parenting, school involvement, and encouragement to succeed. Child Development. 1992;63:1266-1281.

33. Ezpeleta L, Keeler G, Alaatin E, et al. Epidemiology of psychiatric disability in childhood and adolescence. Journal of Child Psychology and Psychiatry and Allied Disciplines. 2001;42(7):901-914.

34. Hartwell S. An examination of racial differences among mentally ill offenders in Massachusetts. Psychiatric Services. 2001;52(2):234-236.

35. Compton WM, Cottler LB, Abdallah AB, et al. Substance dependence and other psychiatric disorders among drug dependent subjects: race and gender correlates. American Joumal on Addictions. 2000;9(2):113-125.

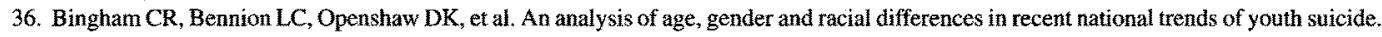
Journal of Adolescence. 1994;17(1):53-71.

37. Kessler R, McGonagle KA, Zhao S, et al. Lifetime and 12-month prevalence of DSM-III-R psychiatric disorders in the US: results from the National Comorbidity Survey. Archives of General Psychiatry. 1994;51:8-19.

38. US Department of Health and Human Services. Mental Health: Culture, Race, Ethnicity Supplement to Mental Health. Report of the Surgeon General. Washington, DC: US Dept of Health \& Human Services; 2001.

39. Alvidrez $\mathbf{J}$. Ethnic variations in mental health attitudes and service use among low-income African American, Latina, and European American young women. Community Mental Health Journal. 1999;35(6):515-530.

40. Leaf PJ, Bruce ML, Tischler GL. The differential effect of attitudes on the use of mental health services. Social Psychiatry. 1986;21(4):187192.

41. Silva de Crane R, Spielberger CD. Attitudes of Hispanic, Black, and Caucasian university students toward mental illness. Hispanic Journal of Behavioral Sciences. 1981;3:241-255.

42. Cauce AM, Domenech-Rodriguez M, Paradise M, et al. Cultural and contextual influences in mental health help seeking: a focus on ethnic minority youth. Journal of Consulting and Clinical Psychology. 2002;70(1):44-55.

43. Offord DR, Boyle MH, Racine YA, et al. Outcome, prognosis, and risk in a longitudinal follow-up study. Joumal of the American Academy of Child \& Adolescent Psychiatry. 1992;31(5):916-923.

44. Bridges GS, Steen S. Racial disparities in official assessments of juvenile offenders: attributional stereotypes as mediating mechanisms. American Sociological Review. 1998;63:554-570.

45. Vega WA, Lopez SR. Priority issues in Latino mental health services research. Mental Health Services Research. 2001;3(4):189-200.

46. McMiller WP, Weisz JR. Help-seeking preceding mental health clinic intake among African-American, Latino, and Caucasian youths. Journal of the American Academy of Child \& Adolescent Psychiatry. 1996;35(8):1086-1094.

47. Rogers KM, Zima B, Powell E, et al. Who is referred to mental health services in the juvenile justice system? Journal of Child and Family Studies. 2001;10(4):485-494.

48. Whaley AL. Ethnic and racial differences in perceptions of dangerousness of persons with mental illness. Psychiatric Services. 1997; 48(10): 1328-1330.

49. Teplin LA, Abram KM, McClelland GM, et al. Psychiatric disorders in youth in juvenile detention. Archives of General Psychiatry. 2002;59(12):1133-1143 